\title{
Assembly of a new family of mercury(II) zwitterionic thiolate complexes from a preformed compound $\left[\mathrm{Hg}(\mathrm{Tab})_{2}\right]\left(\mathrm{PF}_{6}\right)_{2} \quad(\mathrm{Tab}=$ 4-(trimethylammonio)benzenethiolate)
}

Jin-Xiang Chen, Wen-Hua Zhang, Xiao-Yan Tang, Zhi-Gang Ren, Yong Zhang,, Jian-Ping Lang*

Key Laboratory of Organic Synthesis of Jiangsu Province, School of Chemistry and Chemical Engineering, Suzhou University, Suzhou 215006, Jiangsu, P. R.China

State Key Laboratory of Structural Chemistry, Fujian Institute of Research on the Structure of Matter CAS, Fuzhou 350002, Fujian, P. R. China

\section{Table of contents}

Table S1. Hydrogen bond geometry $\left(\AA,^{\circ}\right)$ in $\mathbf{1}, \mathbf{2}, \mathbf{3}, \mathbf{6}, \mathbf{8}, \mathbf{9}$, and 10................................S2-S3

Table S2. The secondary bond distances and angles........................................................S4-S5

Figure S1 Two dimensional network structure along ac plan formed via the hydrogen bonding

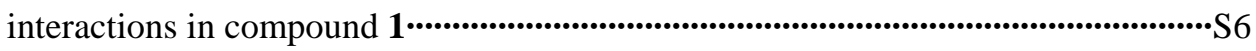

Figure S2 One-dimensional chain structure along $b$ axis formed via the hydrogen bonding

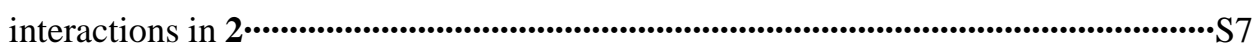

Figure S3 The view of the extended structure of compound 2 looking down along $b$ axis …......S8

Figure S4 Two dimensional network structure extended along ac axis formed via the hydrogen

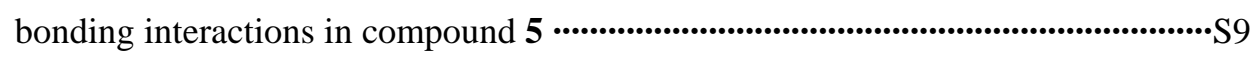

Figure S5 The view of the extended structure of compound $\mathbf{6}$ looking down along $c$ axis........S10

Figure S6 The view of the extended structure of compound $\mathbf{9}$ looking down along $c$ axis $\cdots . . . . . . S 11$

Figure S7 The view of the extended structure of compound $\mathbf{1 0}$ looking down along $b$ axis $\cdots . . . . S 12$ 
Table S1. Hydrogen bond geometry $\left(\AA,^{\circ}\right)$ in $1,2,3,6,8,9$, and 10

$\begin{array}{llll}\text { D-H } \cdots \text { A } & \text { D-H } & \text { H } \cdots \text { A } & \text { D } \cdots \text { A }\end{array}$

Compound 1

$\begin{array}{lllll}\mathrm{C}(8)-\mathrm{H}(8 \mathrm{~A})-\mathrm{F}(2) \# 1 & 0.98 & 2.55 & 3.513(14) & 174.2 \\ \mathrm{C}(8)-\mathrm{H}(8 \mathrm{~B})-\mathrm{F}(4) \# 2 & 0.98 & 2.55 & 3.451(15) & 152.7\end{array}$

$\# 1: x+1, y-1, z ; \# 2:-x+1,-y+1,-z+1$.

Compound 2

$\begin{array}{lllll}\mathrm{C}(6)-\mathrm{H}(6)-\mathrm{S}(1) \# 1 & 0.95 & 2.82 & 3.761(6) & 172.7 \\ \mathrm{C}(9)-\mathrm{H}(9 \mathrm{~A})-\mathrm{N}(4) \# 1 & 0.98 & 2.56 & 3.392(13) & 143.1 \\ \mathrm{C}(23)-\mathrm{H}(23)-\mathrm{S}(1) \# 2 & 0.95 & 2.66 & 3.555(6) & 156.6\end{array}$

$\# 1:-x+2,-y+1,-z ; \# 2: x-1, y, z$.

Compound 4

$\begin{array}{lllll}\mathrm{C}(8)-\mathrm{H}(8 \mathrm{~A})-\mathrm{S}(2) \# 1 & 0.98 & 2.88 & 3.809(5) & 158.9\end{array}$

$\# 1: x-1,-y+3 / 2, z-1 / 2$.

Compound 5

$\begin{array}{lllll}\mathrm{C}(8)-\mathrm{H}(8 \mathrm{C})-\mathrm{I}(2) \# 1 & 0.98 & 2.96 & 3.895(8) & 159.8 \\ \mathrm{C}(8)-\mathrm{H}(8 \mathrm{~A})-\mathrm{I}(2) \# 2 & 0.98 & 3.02 & 3.941(8) & 157.3\end{array}$

$\# 1: x-1, y, z ; \# 2: x-1 / 2,-y+3 / 2, z+1 / 2$.

Compound 6

$\begin{array}{lllll}\mathrm{C}(6)-\mathrm{H}(6)-\mathrm{S}(2) \# 1 & 0.95 & 2.84 & 3.77(2) & 166.3 \\ \mathrm{C}(9)-\mathrm{H}(9 \mathrm{~A})-\mathrm{F}(6) \# 2 & 0.98 & 2.53 & 3.51(4) & 173.7 \\ \mathrm{C}(15)-\mathrm{H}(15)-\mathrm{S}(1) \# 1 & 0.95 & 2.88 & 3.77(2) & 155.8\end{array}$

$\# 1:-x,-y+1 ;-z ; \# 2: x-1, y, z$. 
to be continued for Table S1

Compound 9

C(3)-H(3)-Cl(2)\#1

$0.95 \quad 2.78$

3.723(7)

172.6

$\mathrm{C}(8)-\mathrm{H}(8 \mathrm{C})-\mathrm{Cl}(2) \# 1$

0.98

$3.558(7)$

163.2

$\mathrm{C}(8)-\mathrm{H}(8 \mathrm{~B})-\mathrm{Cl}(5) \# 2$

$0.98 \quad 2.77$

3.656(7)

150.4

C(14)-H(14)-Cl(2)\#3

$0.95 \quad 2.82$

3.756(6)

169.0

C(17)H(17B)-Cl(4)\#4

0.98

2.77

3.717(8)

163.8

$\# 1:-x+1,-y,-z ; \# 2: x+1, y, z ; \# 3:-x+1, y+1 / 2,-z+1 / 2 ; \# 4: x+1,-y+1 / 2, z+1 / 2$.

\section{Compound 10}

$\begin{array}{lllll}\mathrm{C}(5)-\mathrm{H}(5)-\mathrm{Cl}(2) \# 1 & 0.95 & 2.82 & 3.67(7) & 149.2 \\ \mathrm{C}(7)-\mathrm{H}(7 \mathrm{C})-\mathrm{Cl}(2) \# 1 & 0.98 & 2.76 & 3.70(10) & 160.6 \\ \mathrm{C}(9)-\mathrm{H}(9 \mathrm{~B})-\mathrm{Cl}(1) \# 2 & 0.98 & 2.64 & 3.57(7) & 160.0 \\ \mathrm{C}(14)-\mathrm{H}(14)-\mathrm{S}(2) \# 3 & 0.95 & 2.82 & 3.74(6) & 164.0 \\ \mathrm{C}(18)-\mathrm{H}(18 \mathrm{~A})-\mathrm{Cl}(2) \# 4 & 0.98 & 2.72 & 3.63(9) & 154.7 \\ \mathrm{C}(23)-\mathrm{H}(23)-\mathrm{S}(1) \# 5 & 0.95 & 2.69 & 3.63(5) & 170.1 \\ \mathrm{C}(27)-\mathrm{H}(27 \mathrm{~A})-\mathrm{Cl}(4) \# 6 & 0.98 & 2.78 & 3.70(6) & 156.9\end{array}$

$\# 1: 5 / 3+x-y, 4 / 3-y,-1 / 3+z ; \# 2: 1 / 3+x, 2 / 3+y,-1 / 3+z ; \# 3: 4 / 3+x-y, 2 / 3+x, 2 / 3-z ; \# 4: 1-$ $z, 2-y, 1-z ; \# 5: 2-y, 1+x-y, z ; \# 6: 4 / 3-z, 5 / 3-y, 2 / 3-z$. 
Table S2. The secondary bond distances and angles

1

$\mathrm{Hg}(1)-\mathrm{F}(2)$

$\mathrm{S}(1)-\mathrm{Hg}(1)-\mathrm{F}(2)$

(F2)-Hg(1)-F(2A)

3

Hg(1)-S(16D)

Hg(3)-S(5)

Hg(5B)-S(11A)

S(16D)-Hg(1)-S(1)

87.089(14)

107.774(17)

S(16D)-Hg(1)-S(3)

84.830(14)

$\mathrm{S}(1)-\mathrm{Hg}(2)-\mathrm{S}(5)$

S(5)-Hg(3)-S(7)

S(5)-Hg(3)-S(9)

S(7)-Hg(4A)-S(11A)

S(11A)-Hg(5B)-S(13B)

S(11A)-Hg(5B)-S(15B)

S(14B)-Hg(6C)-S(17C)

Hg(6D)-S(16D)-Hg(1)

Hg(2)-S(5)-Hg(3)

Hg(4A)-S(11A)-Hg(5B)

5

Hg(1)-I(1A)

$\mathrm{I}(1 \mathrm{~A})-\mathrm{Hg}(1)-\mathrm{I}(2)$

$\mathrm{S}(1)-\mathrm{Hg}(1)-\mathrm{I}(1 \mathrm{~A})$

S(1B)- Hg(1)-I(1)

Hg(1)-I(1A)-Hg(1A)
85.321(2)

89.088(2)

94.169(2)

77.840(13)

105.058(16)

83.592(14)

91.488(13)

107.718(17)

87.054(14)

100.801(16)

97.258(16)

97.557(16)

3.6170(4)

$79.978(2)$
$\mathrm{S}(1 \mathrm{~A})-\mathrm{Hg}(1)-\mathrm{F}(2)$

$80.741(2)$

$\operatorname{Hg}(2)-S(1)$

3.6148(10)

$\operatorname{Hg}(4 \mathrm{~A})-\mathrm{S}(7)$

3.7903(10)

Hg(6C)-S(14B)

3.9237(9)

$\mathrm{S}(16 \mathrm{D})-\mathrm{Hg}(1)-\mathrm{S}(2) \quad$ 90.756(13)

S(1)-Hg(2)-S(4) 97.093(13)

$\mathrm{S}(1)-\mathrm{Hg}(2)-\mathrm{S}(6)$

101.302(16)

$\mathrm{S}(5)-\mathrm{Hg}(3)-\mathrm{S}(8)$

100.696(13)

S(7)-Hg(4A)-S(10A)

96.304(13)

S(7)-Hg(4A)-S(12A)

107.921(17)

S(11A)-Hg(5B)-S(14B) 83.500(14)

S(14B)-Hg(6C)-S(16C) 87.990(14)

S(14B)-Hg(6C)-S(18C) 126.665(17)

Hg(1)-S(1)-Hg(2) 100.426(16)

Hg(3)-S(7)-Hg(4A) 94.026(15)

Hg(5B)-S(14B)-Hg(6C) 105.661(17)

$\operatorname{Hg}(1)-S(1 B)$

3.1263(4)

$\mathrm{I}(1 \mathrm{~A})-\mathrm{Hg}(1)-\mathrm{I}(1)$

87.716(2)

$\mathrm{S}(1 \mathrm{~B})-\mathrm{Hg}(1)-\mathrm{I}(2)$

89.079(2)

$\mathrm{S}(1 \mathrm{~B})-\mathrm{Hg}(1)-\mathrm{S}(1)$

94.855(2)

Hg(1)-S(1)-Hg(1A) 96.000(2) 


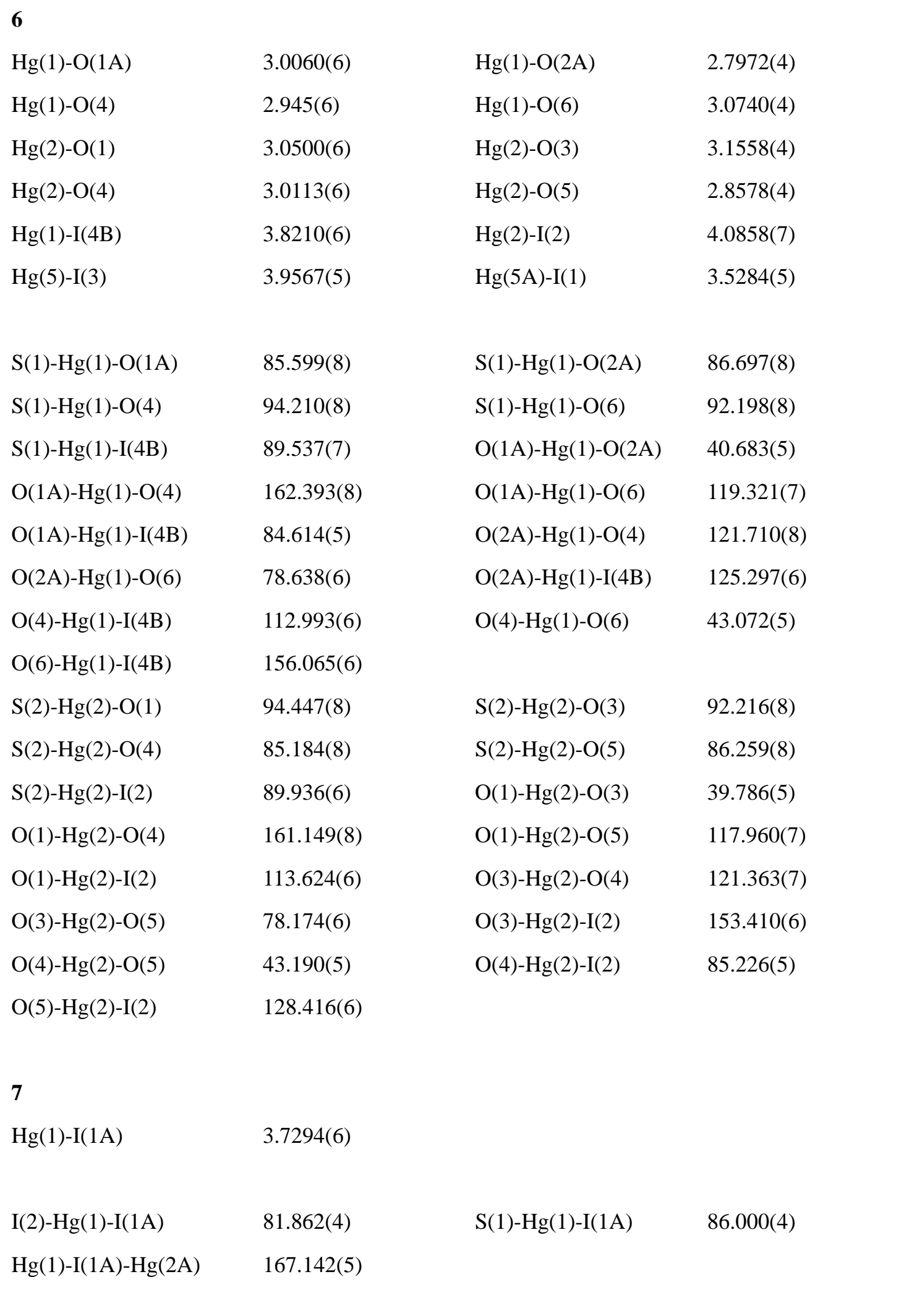




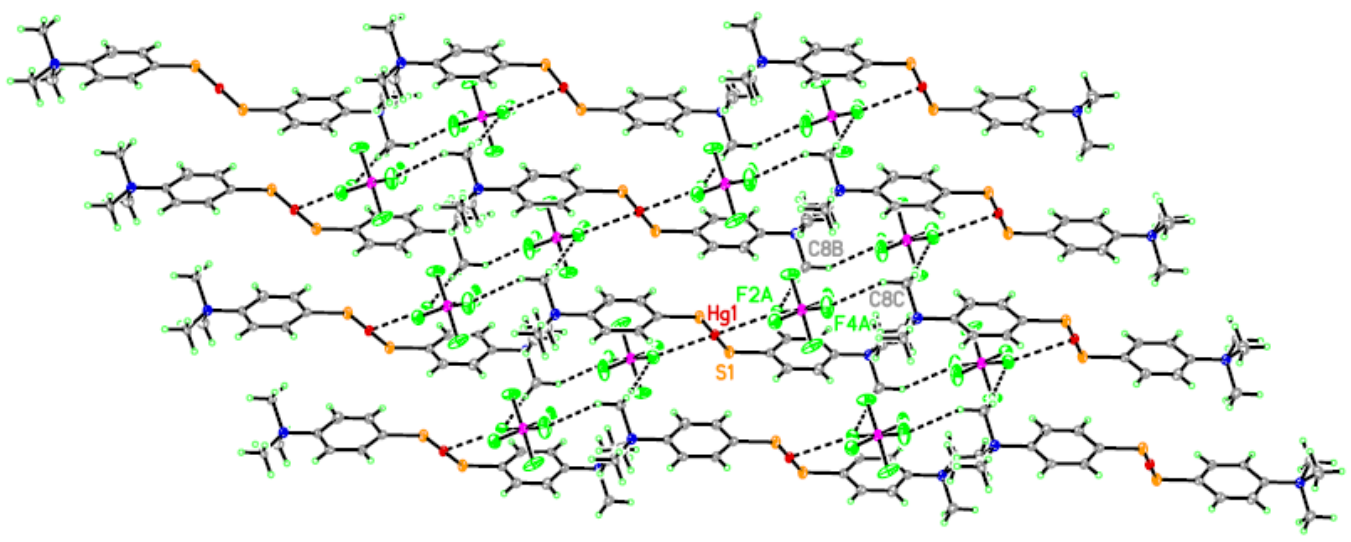

Figure S1 Two dimensional network structure along ac plan formed via the hydrogen bonding interactions in compound $\mathbf{1}$ 


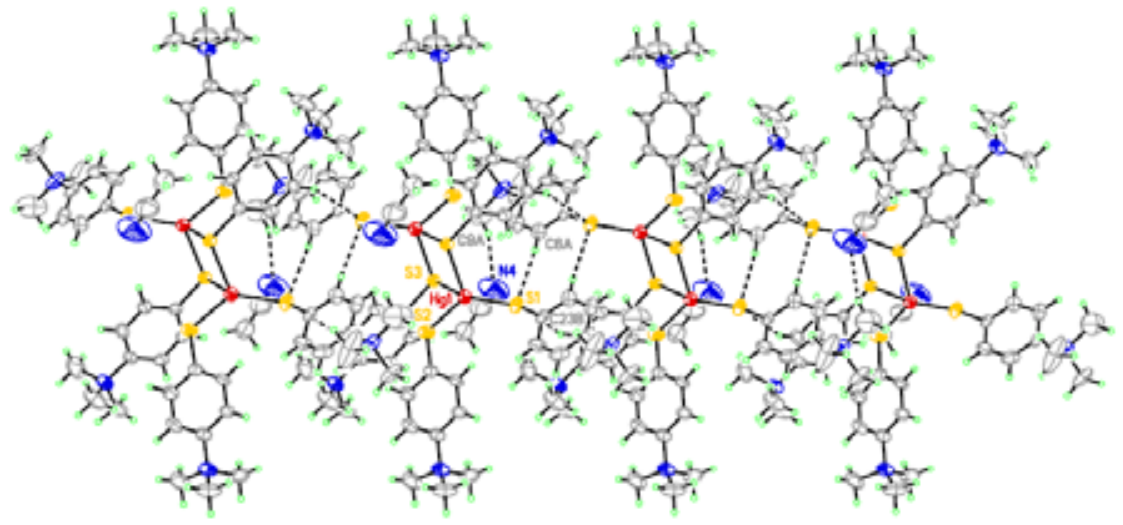

Figure S2. One-dimensional chain structure along $b$ axis formed via the hydrogen bonding interactions in 2 


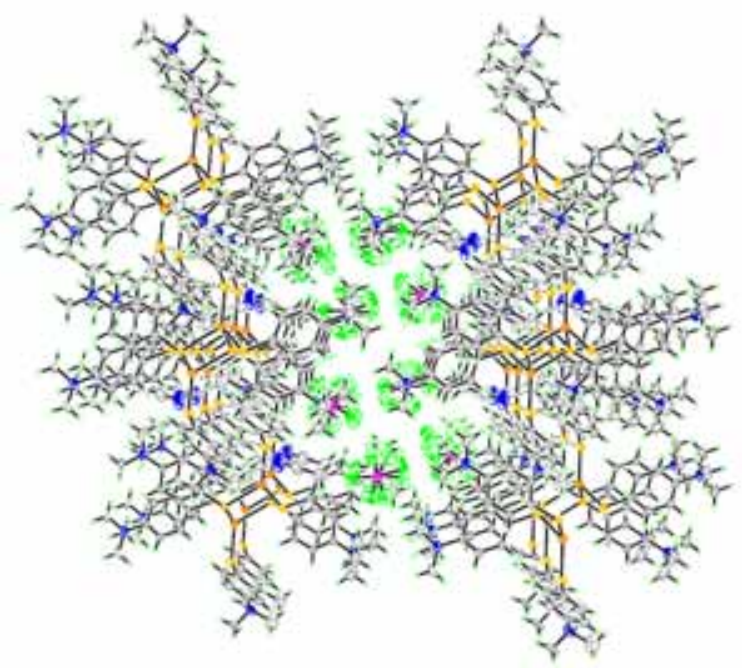

Figure S3. The view of the extended structure of compound 2 looking down along $b$ axis 


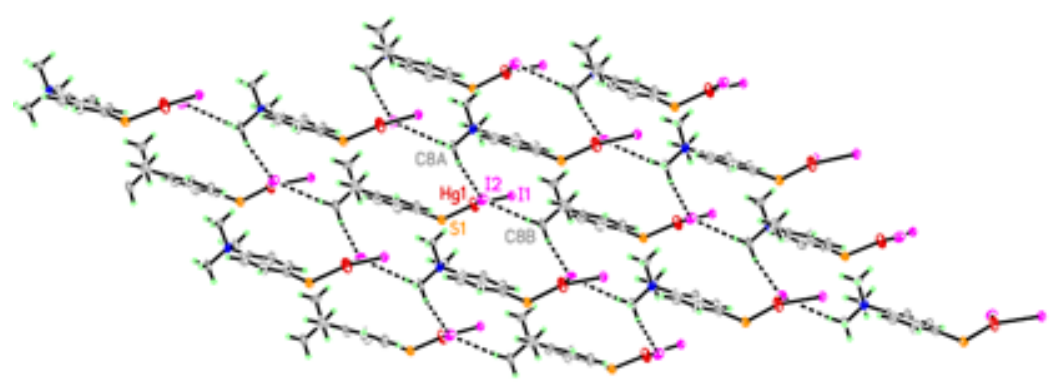

Figure S4. Two dimensional network structure extended along ac axis formed via the hydrogen bonding interactions in $\mathbf{5}$ 


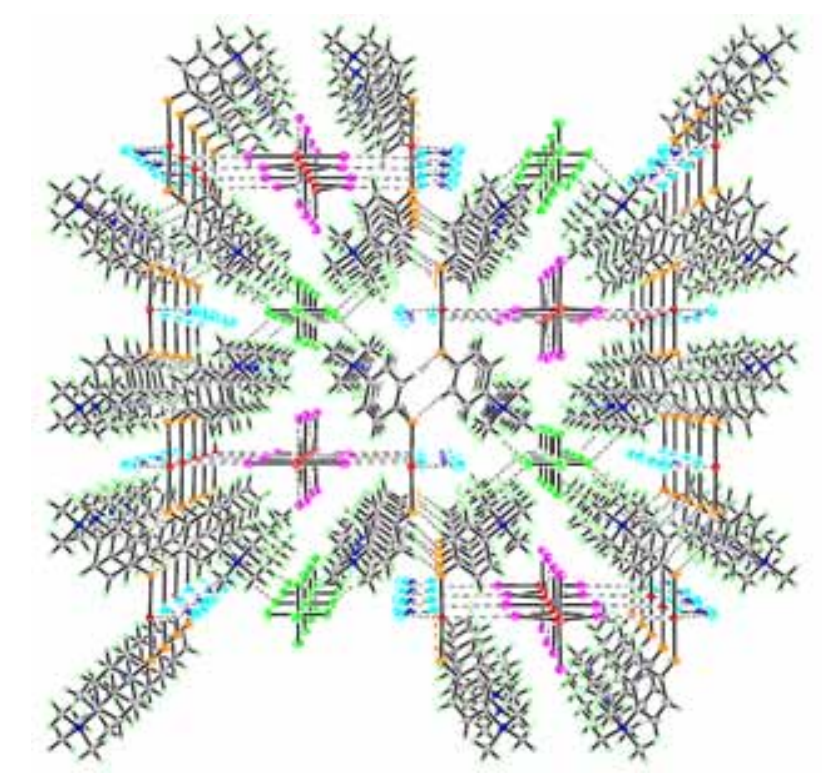

Figure S5. The view of the extended structure of 6 looking down along $c$ axis 


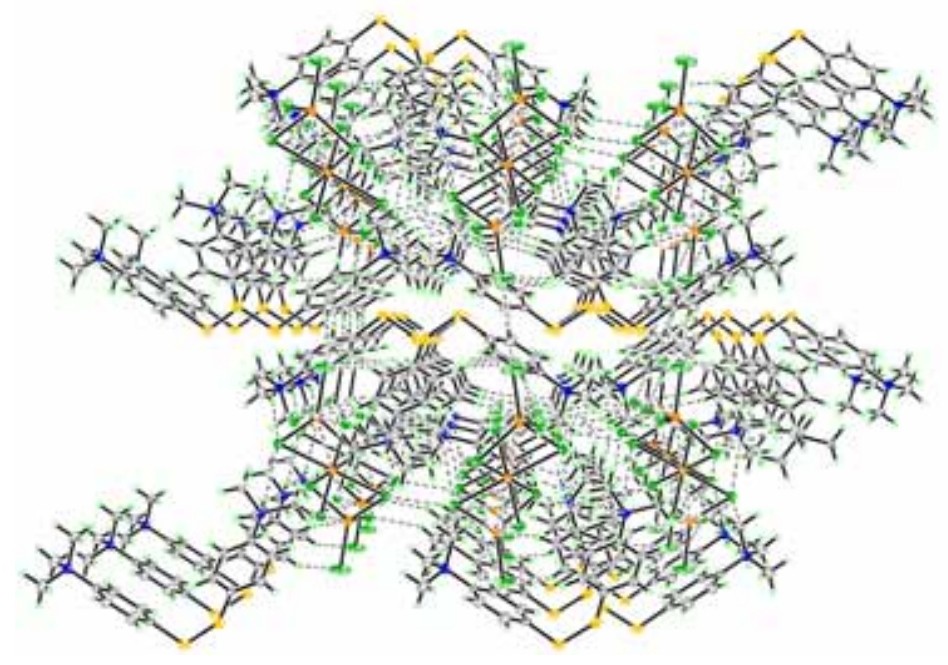

Figure S6. The view of the extended structure of 9 looking down along $c$ axis 


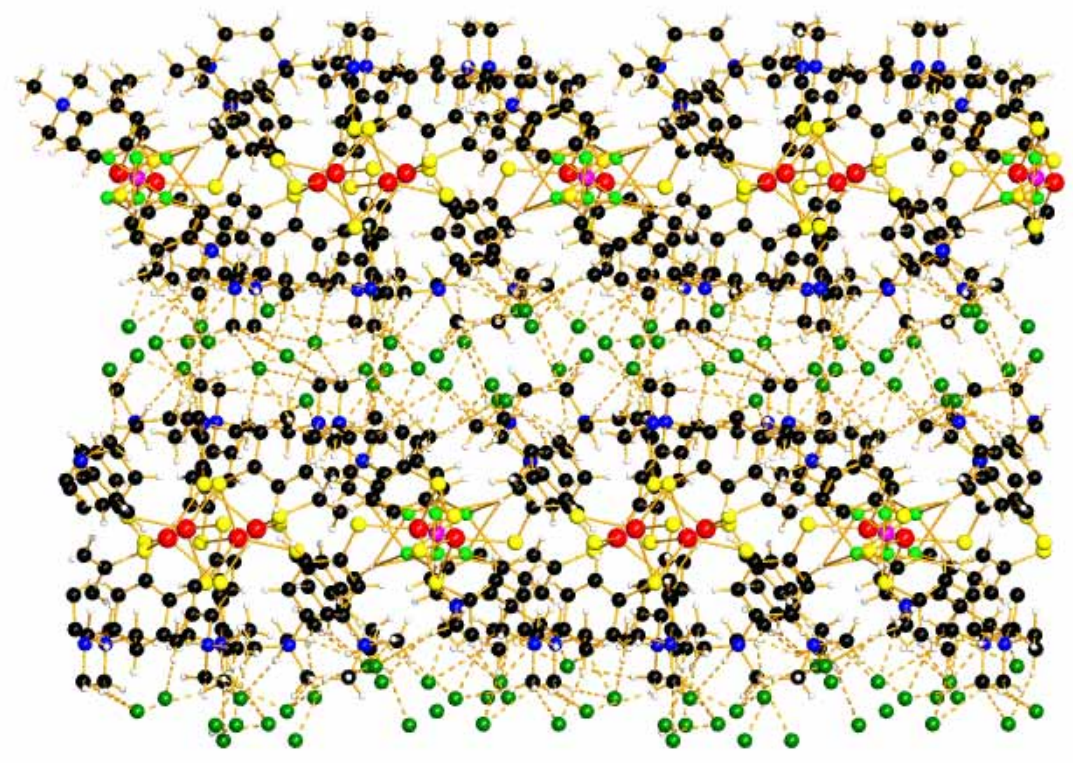

Figure S7. The view of the extended structure of compound $\mathbf{1 0}$ looking down along $b$ axis 